\title{
Clinical characteristics and survival patterns of subsequent sarcoma, breast cancer, and melanoma after childhood cancer in the DCOG-LATER cohort
}

\author{
Jop C. Teepen ${ }^{1,2}$ - Leontien C. Kremer ${ }^{1,2} \cdot$ Margriet van der Heiden-van der Loo ${ }^{3} \cdot$ Wim J. Tissing ${ }^{2,4}$. \\ Helena J. van der Pal ${ }^{2}$. Marry M. van den Heuvel-Eibrink ${ }^{2,5}$. Jacqueline J. Loonen ${ }^{6}$. Marloes Louwerens ${ }^{7}$. \\ Birgitta Versluys $^{8}$. Eline van Dulmen-den Broeder ${ }^{9}$. Otto Visser ${ }^{10}$. John H. Maduro ${ }^{11}$. Flora E. van Leeuwen ${ }^{12}$. \\ Cecile M. Ronckers ${ }^{1,2}$ t the DCOG-LATER Study Group
}

Received: 16 January 2019 / Accepted: 28 June 2019 / Published online: 12 July 2019

(c) The Author(s) 2019

\begin{abstract}
Purpose Childhood cancer survivors are at increased risk of developing subsequent malignant neoplasms (SMNs). We compared survival and clinical characteristics of survivors with SMNs (sarcoma, breast cancer, or melanoma) and a populationbased sample of similar first malignant neoplasm (FMN) patients.

Methods We assembled three case series of solid SMNs observed in a cohort of 5-year Dutch childhood cancer survivors diagnosed 1963-2001 and followed until 2014: sarcoma $(n=45)$, female breast cancer $(n=41)$, and melanoma $(n=17)$. Each SMN patient was sex-, age-, and calendar year-matched to 10 FMN patients in the population-based Netherlands Cancer Registry. We compared clinical and histopathological characteristics by Fisher's exact tests and survival by multivariable Cox regression and competing risk regression analyses.

Results Among sarcoma-SMN patients, overall survival [hazard ratio (HR) 1.88, 95\% confidence interval (CI) 1.23-2.87] and sarcoma-specific mortality (HR 1.91, 95\% CI 1.16-3.13) were significantly worse compared to sarcoma-FMN patients (foremost for soft-tissue sarcoma), with 15-year survival rates of $30.8 \%$ and $61.6 \%$, respectively. Overall survival did not significantly differ for breast-SMN versus breast-FMN patients (HR 1.14, 95\% CI 0.54-2.37), nor for melanoma-SMN versus melanoma-FMN patients (HR 0.71, 95\% CI 0.10-5.00). No significant differences in tumor characteristics were observed between breast-SMN and breast-FMN patients. Breast-SMN patients were treated more often with mastectomy without radiotherapy/chemotherapy compared to breast-FMN patients (17.1\% vs. $5.6 \%)$.

Conclusions Survival of sarcoma-SMN patients is worse than sarcoma-FMN patients. Although survival and tumor characteristics appear similar for breast-SMN and breast-FMN patients, treatment differs; breast-SMN patients less often receive breast-conserving therapy. Larger studies are necessary to substantiate these exploratory findings.
\end{abstract}

Keywords Childhood cancer survivors · Subsequent malignant neoplasm $\cdot$ Survival $\cdot$ Epidemiology $\cdot$ Long-term complications

Flora E. van Leeuwen and Cecile M. Ronckers have contributed equally to this work.

The members of the DCOG-LATER Study Group are listed in the acknowledgements.

Electronic supplementary material The online version of this article (https://doi.org/10.1007/s10552-019-01204-z) contains supplementary material, which is available to authorized users.

Jop C. Teepen

J.C.Teepen@prinsesmaximacentrum.nl

Extended author information available on the last page of the article

\section{Introduction}

Survivors of childhood cancer are at increased risk of developing long-term health problems even decades after treatment [1-4]. Subsequent malignant neoplasms (SMNs) are among the most serious adverse conditions that childhood cancer survivors may develop and represent a major cause of morbidity and mortality [5-9].

Several studies have addressed differences in prognostic, clinical, and/or histopathological characteristics of specific types of solid SMNs after childhood, adolescent, and young 
adult cancers and comparable first malignant neoplasms (FMNs) in the general population [10-19]. Since the majority of these type of studies focused on breast-SMN, most were conducted in Hodgkin lymphoma or other adolescent and young adult (AYA) cancer survivors. Only few studies have specifically focused on young cancer survivors $[12,14$, 17] and few have compared cause-specific survival between patients with specific types of SMNs following cancer at a young age and comparable FMN patients [14, 16].

Therefore, we examined clinical characteristics, overall survival, and cause-specific survival of patients in the Dutch Childhood Cancer Oncology Group-Long-Term Effects After Childhood Cancer (DCOG-LATER) cohort who developed SMNs of sarcoma, breast, or melanoma and compared those outcomes with matched patients having similar FMNs in the general population, based on the Netherlands Cancer Registry (NCR). We hypothesized that survival of SMNs may be different from survival of FMNs for several reasons: earlier detection due to surveillance efforts among survivors (favorable), variability of tumor characteristics due to different pathogenesis (favorable or unfavorable), limited treatment options due to previous childhood cancer treatment (unfavorable), and a higher frequency of other potentially lethal long-term chronic health conditions (unfavorable).

\section{Methods}

\section{Subsequent solid malignant neoplasms in the DCOG-LATER cohort}

The DCOG-LATER cohort includes 6,165 five-year childhood cancer survivors diagnosed before the age of 18 years in one of the seven Dutch pediatric oncology and stem cell transplant centers between 1 January 1963 and 31 December 2001. Detailed information on childhood cancer diagnosis and treatment was collected and incident SMNs were obtained via various methods, as previously described [8]. This particular study included cohort members with SMNs (referred to as SMN patients hereafter) diagnosed from 1989 through 2014 that were verified by the NCR (populationbased $\geq 1989$ ) [20]. We designed three case series of most prevalent invasive subsequent solid SMNs observed in the DCOG-LATER cohort: sarcoma, female breast cancer, and melanoma. Eligible morphology/topography codes are presented in Supplementary Table 1. In an earlier report on the current cohort, sarcoma, female breast cancer, and melanoma were shown to represent $20 \%, 20 \%$, and $4 \%$, respectively, of the total number of solid SMNs [8]. To avoid misclassification and bias, we excluded patients for whom the respective SMN of inclusion was a third tumor preceded by an intervening second malignancy treated with radiotherapy and/or chemotherapy.

\section{Population-based first malignant neoplasms (FMN)}

Each of the SMN patients in the three case series (sarcoma, female breast cancer, and melanoma) from the DCOGLATER cohort was matched to 10 comparison patients with FMNs from the population-based NCR (referred to as FMN patients hereafter), based on sex, age at cancer diagnosis, calendar year of cancer diagnosis \pm 3 years, and for sarcomas also on primary site (bone/soft tissue). When $>10$ comparison patients fulfilled these criteria, the 10 patients closest to incidence date of the corresponding case were selected. When less than the 10 comparison FMN patients from the cancer registry fulfilled these criteria for a specific index SMN patient, the matching criterion for age was widened until 10 eligible comparison FMN patients were identified. That is, the FMN comparison group was supplemented with cancer patients closest in age at their respective FMN cancer diagnosis to the index SMN case diagnosis, but within the same diagnosis calendar year defined by the index SMN case. Sampled comparison patients were excluded from serving as FMN patients if they had a history of cancer, with the exception of basal cell carcinoma.

\section{Data on prognostic, clinical, and histopathological characteristics}

The following SMN/FMN characteristics were obtained from the NCR: morphology, stage, grade, treatment (for all three cancer sites yes/no indicators for surgery, chemotherapy, and radiotherapy and for breast cancer additionally yes/no indicators for mastectomy, breast-conserving surgery, and hormonal therapy), and vital status. For patients with breast cancer, information on receptor status was also retrieved. No genetic data were available. Patients were followed until date of death, date of emigration, or through $12 / 31 / 2016$, whichever occurred first. Coded cause of death information for deceased SMN/FMN patients was obtained by linkage with Statistics Netherlands. We categorized cause of death into cancer of interest-related deaths and death due to other causes. Data collection for the study was exempted from review by the Institutional Review Boards of all participating centers and all data were processed and analyzed anonymously.

\section{Statistical analyses}

We compared clinical and histopathological characteristics for SMN and FMN patients by Fisher's exact tests. Overall survival was calculated using Kaplan-Meier methods and represented by Kaplan-Meier curves. For cause-specific mortality, cumulative incidences functions (probability of 
death due to the specific cause) were estimated. When evaluating cancer of interest-related deaths, deaths due to other causes were treated as a competing risk and vice versa. We used Cox proportional hazard regression analyses to test differences in overall survival between the SMN and FMN groups in univariable models with patient group (SMN vs. FMN) as an independent variable and in multivariable models with additional factors hypothesized to influence a difference in survival between the SMN and FMN groups. For sarcoma, morphology was one of the factors we adjusted for in the analyses. For any sarcoma, we grouped morphology into three groups based on similarity in survival estimates reported in literature: (1) osteosarcoma/rhabdomyosarcoma; (2) chondrosarcoma; (3) other or unspecified. Proportionality of hazards for each variable was evaluated by adding interaction terms with follow-up time. Fine and Gray competing risk models were used to test differences between SMN and FMN patients with respect to mortality due to the cancer of interest and mortality from other causes [21]. All analyses were performed with Stata 14 software (StataCorp. 2015. Stata Statistical Software: Release 14. College Station, TX: StataCorp LP).

\section{Results}

\section{Sarcoma-comparison of clinical and histopathological characteristics}

Of 46 eligible patients with subsequent sarcoma (sarcomaSMN) after childhood cancer, one patient was excluded because of a SMN diagnosis (acute lymphoblastic leukemia (ALL); treatment with radiotherapy and chemotherapy) prior to developing a sarcoma as third malignancy. We also excluded one comparison patient in the first primary sarcoma (sarcoma-FMN) group, who was erroneously selected for inclusion, despite a history of a prior cancer. In total, we included 45 sarcoma-SMN patients and 449 sarcomaFMN patients. Among sarcoma-SMN patients, $30.4 \%$ also had a sarcoma as childhood cancer diagnosis, $8.7 \%$ had a retinoblastoma as childhood cancer diagnosis, and $60.9 \%$ had previous radiotherapy treatment to the sarcoma-SMN region (Supplementary Table 2).

Several differences were observed in the morphology of sarcoma-SMNs and sarcoma-FMNs. Twenty-seven percent of sarcoma-SMN patients had osteosarcoma, versus $12.9 \%$ of sarcoma-FMN patients (Table 1$)(p=0.022$, not shown). In contrast, none of the sarcoma-SMN patients had Ewing sarcoma, while this was the morphology of $13.1 \%$ of sarcoma-FMN patients. Fibrosarcoma was much less common among sarcoma-SMN patients $(2.2 \%)$ than among sarcoma-FMN patients $(16.5 \%)(p=0.008$, not shown), with dermatofibrosarcomas protuberans being the largest contributor of fibrosarcomas among FMN patients (62 cases, 13.8\%). Remarkably, no dermatofibrosarcomas protuberans occurred among sarcoma-SMN patients. Among the minority with a known tumor grade (34.4\%), sarcoma-SMN patients were more likely to have a high tumor grade than sarcoma-FMN patients (SMN 60.0\% vs. FMN $26.0 \%$ poor/high grade and SMN $10.0 \%$ vs. FMN $2.0 \%$ undifferentiated/anaplastic grade for sarcoma-SMN and sarcoma-FMN patients, respectively; $p=0.002$ ). We did not observe significant differences in stage distribution or treatments received.

\section{Sarcoma-overall and cause-specific survival}

Median follow-up time was 3.6 years ( $\max 34.0$ years) for sarcoma-SMN patients and 7.5 years ( $\max 28.0$ years) for sarcoma-FMN patients. At the end of follow-up, $62.2 \%$ of sarcoma-SMN patients were deceased compared to $33.2 \%$ of sarcoma-FMN patients (Table 1). Because dermatofibrosarcoma protuberans behaves in many respects as a benign tumor and has very high survival rates [22], we excluded those patients ( $n=62$, all sarcoma-FMN patients) from all survival analyses. Furthermore, we excluded Ewing sarcoma ( $n=59$, all sarcoma-FMN patients) and Kaposi sarcoma ( $n=19,18$ sarcoma-FMN patients and 1 sarcoma-SMN patient) from all survival analyses, because those sarcoma types are rare to occur as SMN. Survival rates after 5, 10, and 15 years were $44.9 \%, 38.5 \%$, and $30.8 \%$, respectively, among sarcoma-SMN patients, considerably lower than those among sarcoma-FMN patients with $67.4 \%, 63.1 \%$, and $61.6 \%$, respectively (Table 2, Fig. 1a). Overall survival was significantly worse among sarcoma-SMN patients (multivariable HR 1.88; 95\% CI 1.28-2.87) (Table 3, Fig. 1). Sarcoma-specific mortality was also significantly higher in sarcoma-SMN patients compared to sarcoma-FMN patients (15-year mortality $66.0 \%$ vs. $34.5 \%$ and multivariable HR 1.91; 95\% CI 1.16-3.13, Tables 2 and 3). Mortality due to other causes after 5,10 , and 15 years was very low in all groups $(<3 \%)$.

Stratified by type of sarcoma, survival differences between SMN and FMN patients seemed to be greater for soft-tissue sarcoma (multivariable HR 2.31; 95\% CI 1.37-3.89) than for bone sarcoma (multivariable HR 1.21; 95\% CI 0.57-2.53) (Supplementary Table 3, Supplementary Fig. 1). In analyses per specific sarcoma morphology type, we found no difference in survival between osteosarcomaSMN and FMN patients (multivariable HR 1.02; 95\% CI 0.45-2.34), but we found a statistically significant difference between synovial sarcoma SMN and FMN patients (multivariable HR 7.82; 95\% CI 1.41-43.35) (not shown). For other specific sarcoma morphology types, numbers were too small to make meaningful comparisons. 
Table 1 Characteristics of patients with sarcomas and melanomas as a subsequent malignant neoplasm (SMN) and matched patients with sarcomas and melanomas as a first malignant neoplasm (FMN)

\begin{tabular}{|c|c|c|c|c|c|c|c|c|c|c|}
\hline & \multicolumn{4}{|c|}{ Sarcoma } & \multirow[t]{3}{*}{$p$ value } & \multicolumn{4}{|c|}{ Melanoma } & \multirow[t]{3}{*}{$p$ value } \\
\hline & \multicolumn{2}{|c|}{$\begin{array}{l}\text { DCOG-LATER } \\
\text { cohort SMN } \\
(n=45)\end{array}$} & \multicolumn{2}{|c|}{$\begin{array}{l}\text { Cancer registry } \\
\text { FMN } \\
(n=449)^{\mathrm{a}}\end{array}$} & & \multicolumn{2}{|c|}{$\begin{array}{l}\text { DCOG-LATER } \\
\text { cohort SMN } \\
(n=17)\end{array}$} & \multicolumn{2}{|c|}{$\begin{array}{l}\text { Cancer registry } \\
\text { FMN } \\
(n=170)\end{array}$} & \\
\hline & $N$ & $\%$ & $N$ & $\%$ & & $N$ & $\%$ & $N$ & $\%$ & \\
\hline Age at diagnosis ${ }^{\mathrm{b}}$ & & & & & 0.924 & & & & & 1.000 \\
\hline$<30$ years & 35 & 77.8 & 335 & 74.6 & & 6 & 35.3 & 60 & 35.3 & \\
\hline 30-39 years & 8 & 17.8 & 83 & 18.5 & & 6 & 35.3 & 60 & 35.3 & \\
\hline $40+$ years & 2 & 4.4 & 31 & 6.9 & & 5 & 29.4 & 50 & 29.4 & \\
\hline $\operatorname{Sex}^{b}$ & & & & & 1.000 & & & & & 1.000 \\
\hline Male & 25 & 55.6 & 249 & 55.5 & & 6 & 35.3 & 60 & 35.3 & \\
\hline Female & 20 & 44.4 & 200 & 44.5 & & 11 & 64.7 & 110 & 64.7 & \\
\hline Incidence year ${ }^{\mathrm{b}}$ & & & & & 0.909 & & & & & 1.000 \\
\hline 1989-1999 & 12 & 26.7 & 129 & 28.7 & & 3 & 17.7 & 29 & 17.1 & \\
\hline 2000-2009 & 25 & 55.6 & 249 & 55.5 & & 8 & 47.1 & 81 & 47.7 & \\
\hline 2010-2014 & 8 & 17.8 & 71 & 15.8 & & 6 & 35.3 & 60 & 35.3 & \\
\hline Morphology sarcoma & & & & & $\mathrm{NE}$ & & & & & \\
\hline Osteosarcoma & 12 & 26.7 & 58 & 12.9 & & & & & & \\
\hline Chondrosarcoma & 4 & 8.9 & 57 & 12.7 & & & & & & \\
\hline Ewing sarcoma & 0 & 0.0 & 59 & 13.1 & & & & & & \\
\hline Rhabdomyosarcoma & 3 & 6.7 & 23 & 5.1 & & & & & & \\
\hline Leiomyosarcoma & 4 & 8.9 & 22 & 4.9 & & & & & & \\
\hline Fibrosarcoma & 1 & 2.2 & 74 & 16.5 & & & & & & \\
\hline Malignant fibrous histiocytoma & 2 & 4.4 & 12 & 2.7 & & & & & & \\
\hline Malignant peripheral nerve sheath tumor & 3 & 6.7 & 23 & 5.1 & & & & & & \\
\hline Liposarcoma & 3 & 6.7 & 19 & 4.2 & & & & & & \\
\hline Synovial sarcoma & 3 & 6.7 & 24 & 5.4 & & & & & & \\
\hline Kaposi sarcoma & 1 & 2.2 & 18 & 4.0 & & & & & & \\
\hline Other or unspecified bone & 1 & 2.2 & 19 & 4.2 & & & & & & \\
\hline Other or unspecified soft tissue ${ }^{d}$ & 8 & 17.8 & 41 & 9.1 & & & & & & \\
\hline Morphology melanoma & & & & & & & & & & 0.335 \\
\hline Nodular & & & & & & 1 & 5.9 & 20 & 11.8 & \\
\hline Superficial spreading & & & & & & 15 & 88.2 & 117 & 68.8 & \\
\hline Other or unspecified & & & & & & 1 & 5.9 & 33 & 19.4 & \\
\hline Stage $^{e}$ & & & & & 0.977 & & & & & 1.000 \\
\hline I & 12 & 57.1 & 106 & 50.2 & & 13 & 76.5 & 120 & 73.6 & \\
\hline II & 5 & 23.8 & 53 & 25.1 & & 3 & 17.7 & 28 & 17.2 & \\
\hline III & 0 & 0.0 & 7 & 3.3 & & 1 & 5.9 & 15 & 9.2 & \\
\hline IV & 4 & 19.1 & 45 & 21.3 & & 0 & 0.0 & 0 & 0.0 & \\
\hline Unknown & 24 & & 238 & & & 0 & & 7 & & \\
\hline Grade $^{e}$ & & & & & 0.002 & & & & & $\mathrm{NE}$ \\
\hline Well/low grade & 4 & 20.0 & 79 & 52.7 & & 0 & 0.0 & 0 & 0.0 & \\
\hline Moderate/intermediate & 2 & 10.0 & 29 & 19.3 & & 0 & 0.0 & 0 & 0.0 & \\
\hline Poor/high & 12 & 60.0 & 39 & 26.0 & & 0 & 0.0 & 0 & 0.0 & \\
\hline Undifferentiated/anaplastic & 2 & 10.0 & 3 & 2.0 & & 0 & 0.0 & 0 & 0.0 & \\
\hline Unknown & 25 & & 299 & & & 17 & & 170 & & \\
\hline Surgery & & & & & 0.256 & & & & & 0.382 \\
\hline No & 13 & 28.9 & 95 & 21.2 & & 1 & 5.9 & 4 & 2.4 & \\
\hline Yes & 32 & 71.1 & 354 & 78.8 & & 16 & 94.1 & 166 & 97.7 & \\
\hline Radiotherapy & & & & & 0.716 & & & & & 1.000 \\
\hline
\end{tabular}


Table 1 (continued)

\begin{tabular}{|c|c|c|c|c|c|c|c|c|c|c|}
\hline & \multicolumn{4}{|c|}{ Sarcoma } & \multirow[t]{3}{*}{$p$ value } & \multicolumn{4}{|c|}{ Melanoma } & \multirow[t]{3}{*}{$p$ value } \\
\hline & \multicolumn{2}{|c|}{$\begin{array}{l}\text { DCOG-LATER } \\
\text { cohort SMN } \\
(n=45)\end{array}$} & \multicolumn{2}{|c|}{$\begin{array}{l}\text { Cancer registry } \\
\text { FMN } \\
(n=449)^{\mathrm{a}}\end{array}$} & & \multicolumn{2}{|c|}{$\begin{array}{l}\text { DCOG-LATER } \\
\text { cohort SMN } \\
(n=17)\end{array}$} & \multicolumn{2}{|c|}{$\begin{array}{l}\text { Cancer registry } \\
\text { FMN } \\
(n=170)\end{array}$} & \\
\hline & $N$ & $\%$ & $N$ & $\%$ & & $N$ & $\%$ & $N$ & $\%$ & \\
\hline No & 33 & 73.3 & 341 & 76.0 & & 17 & 100 & 168 & 98.8 & \\
\hline Yes & 12 & 26.7 & 108 & 24.1 & & 0 & 0.0 & 2 & 1.2 & \\
\hline Chemotherapy & & & & & 0.632 & & & & & NA \\
\hline No & 26 & 57.8 & 277 & 61.7 & & 17 & 100 & 170 & 100 & \\
\hline Yes & 19 & 42.2 & 172 & 38.3 & & 0 & 0.0 & 0 & 0.0 & \\
\hline Treatment category & & & & & 0.165 & & & & & 0.493 \\
\hline No treatment & 6 & 13.3 & 23 & 5.1 & & 1 & 5.9 & 4 & 2.4 & \\
\hline Surgery only & 16 & 35.6 & 199 & 44.3 & & 16 & 94.1 & 164 & 96.5 & \\
\hline Surgery $+\mathrm{RT}$ and/or CT & 16 & 35.6 & 155 & 34.5 & & 0 & 0.0 & 2 & 1.2 & \\
\hline RT and/or CT only & 7 & 15.6 & 72 & 16.0 & & 0 & 0.0 & 0 & 0.0 & \\
\hline Vital status at end of follow-up & & & & & $<0.001$ & & & & & 0.685 \\
\hline Alive & 17 & 37.8 & 300 & 66.8 & & 15 & 88.2 & 153 & 90.0 & \\
\hline Deceased & 28 & 62.2 & 149 & 33.2 & & 2 & 11.8 & 17 & 10.0 & \\
\hline
\end{tabular}

$C T$ chemotherapy, $F M N$ first malignant neoplasm, $N E$ not estimable, $R T$ radiotherapy, $S M N$ subsequent malignant neoplasm

${ }^{a} 1$ control was excluded, because this patient appeared to have had a prior malignancy

${ }^{\mathrm{b}}$ Matching factors

${ }^{\mathrm{c}}$ Included 1 unspecified bone sarcoma among sarcoma-SMN patients

${ }^{\mathrm{d}}$ Included 2 each with unspecified soft-tissue sarcoma and giant cell sarcoma, 1 each with spindle cell sarcoma, round cell sarcoma, hemangiosarcoma, and hemangiopericytoma among sarcoma-SMN patients

${ }^{\mathrm{e}}$ Percentages and $p$ values for stage and grade are based on subjects with known information only

\section{Breast cancer-comparison of clinical and histopathological characteristics}

Of 42 eligible patients with subsequent female breast cancer (breast-SMN) after childhood cancer, one was excluded because of radiotherapy treatment for an intervening meningioma prior to breast cancer diagnosis. We included 41 breast-SMN patients and 410 matched first primary female breast cancer (breast-FMN) patients. Of all breast-SMN cases, $17.1 \%$ occurred after a Hodgkin lymphoma diagnosis and $40.0 \%$ after a sarcoma diagnosis (Supplementary Table 2). Almost one-third of the breast-SMN patients (31.7\%) had no prior radiotherapy and also nearly onethird $(31.7 \%)$ had prior radiotherapy to the region where the breast cancer developed (26.8\% with chest radiotherapy and $4.9 \%$ with total body irradiation). More than half of the breast-SMN patients $(53.7 \%)$ had prior anthracycline treatment (Supplementary Table 2).

Table 4 shows tumor and breast cancer treatment characteristics of the breast-SMN and breast-FMN patients. The proportion of patients with infiltrating ductal carcinoma was the same in the breast-SMN (87.8\%) and FMN $(87.3 \%)$ groups, as were the distributions of stage and grade. Patients in the breast-SMN group were more likely to have been treated without surgery (SMN $9.8 \%$ vs. FMN $3.2 \%$ ), but markedly more likely to have received mastectomy (SMN 63.4\% vs. FMN 49.3\%) than breast-FMN patients $(p=0.024)$. Breast-SMN patients were less likely, however, to have received radiotherapy (SMN 51.2\% vs. FMN $71.7 \%, p=0.011)$ and chemotherapy (SMN 58.5\% vs. FMN $76.3 \%, p=0.011$ ) compared to breast-FMN patients. No significant differences were observed in hormone receptor status single or combined, i.e., triple negative, and receipt of hormonal therapy between breast-SMN and breast-FMN patients. When evaluating combinations of treatments, a higher proportion of breast-SMN patients received mastectomy without $\mathrm{RT}$ or $\mathrm{CT}$ compared to breast-FMN patients (SMN 17.1\% vs. FMN 5.6\%). Also, a lower proportion of breast-SMN patients was treated with breast-conserving surgery with radiotherapy and/or chemotherapy (SMN $26.8 \%$ vs. FMN 46.6\%). In both groups, the majority of patients who did not have surgery for breast cancer had stage IV disease (SMN 75.0\% vs. FMN 84.6\%). For at least three of seven (42.9\%) breast-SMN patients with mastectomy without radiotherapy or chemotherapy ( \pm hormonal therapy), there was an indication that prior childhood cancer treatment limited breast cancer treatment options. One breast-SMN patient received a mastectomy 
Table 2 Overall survival and cause-specific mortality of subsequent malignant neoplasm and matched first malignant neoplasm cohorts

\begin{tabular}{|c|c|c|c|c|c|c|}
\hline & \multicolumn{2}{|l|}{ Sarcoma $^{\mathrm{a}}$} & \multicolumn{2}{|l|}{ Breast cancer $^{\mathrm{b}}$} & \multicolumn{2}{|l|}{ Melanoma } \\
\hline & $\operatorname{SMN}(n=45)$ & $\operatorname{FMN}(n=449)^{\mathrm{c}}$ & $\operatorname{SMN}(n=41)$ & $\mathrm{FMN}(n=410)$ & $\operatorname{SMN}(n=17)$ & $\mathrm{FMN}(n=170)$ \\
\hline & $\%(95 \% \mathrm{CI})$ & $\%(95 \% \mathrm{CI})$ & $\%(95 \% \mathrm{CI})$ & $\%(95 \% \mathrm{CI})$ & $\%(95 \% \mathrm{CI})$ & $\%(95 \% \mathrm{CI})$ \\
\hline 5-year overall survival & $44.9(29.9-58.9)$ & $67.4(61.8-72.3)$ & $93.8(77.4-98.4)$ & $88.5(84.7-91.4)$ & $94.1(65.0-99.2)$ & $93.4(88.4-96.3)$ \\
\hline 10-year overall survival & $38.5(23.7-53.2)$ & $63.1(57.3-68.3)$ & $73.6(52.0-86.6)$ & $79.9(74.8-84.1)$ & $82.4(42.6-95.7)$ & $88.9(82.3-93.2)$ \\
\hline 15 -year overall survival & $30.8(16.5-46.3)$ & $61.6(55.6-67.1)$ & $63.1(34.9-81.8)$ & $77.4(71.5-82.2)$ & $82.4(42.6-95.7)$ & $87.2(79.5-92.2)$ \\
\hline \multicolumn{7}{|l|}{5 -year mortality ${ }^{\mathrm{d}}$} \\
\hline Cancer of interest $\mathrm{e}^{\mathrm{e}}$ & $51.6(35.7-65.3)$ & $30.8(25.6-36.1)$ & $6.2(1.1-17.9)$ & $10.8(7.8-14.3)$ & 0.0 & $5.5(2.7-9.7)$ \\
\hline Other causes $^{\mathrm{f}}$ & $2.4(0.2-11.0)$ & $0.7(0.1-2.2)$ & 0.0 & 0.0 & 0.0 & $0.6(0.1-3.0)$ \\
\hline \multicolumn{7}{|l|}{10 -year mortality ${ }^{\mathrm{d}}$} \\
\hline Cancer of interest $\mathrm{e}^{\mathrm{e}}$ & $58.2(41.2-71.8)$ & $33.9(28.5-39.4)$ & $22.7(9.0-40.0)$ & $17.2(13.2-21.7)$ & $12.5(0.7-42.3)$ & $10.0(5.6-15.8)$ \\
\hline Other causes ${ }^{\mathrm{f}}$ & $2.4(0.2-11.0)$ & $1.9(0.7-4.2)$ & $3.8(0.3-16.1)$ & $0.7(0.1-3.6)$ & 0.0 & $0.6(0.1-3.0)$ \\
\hline \multicolumn{7}{|l|}{15 -year mortality ${ }^{\mathrm{d}}$} \\
\hline Cancer of interest ${ }^{\mathrm{e}}$ & $66.0(47.6-79.3)$ & $34.5(29.0-40.0)$ & $33.2(12.2-56.1)$ & $19.8(15.0-25.1)$ & $12.5(0.7-42.3)$ & $10.0(5.6-15.8)$ \\
\hline Other causes ${ }^{\mathrm{f}}$ & $2.4(0.2-11.0)$ & $1.9(0.7-4.2)$ & $3.8(0.3-16.1)$ & $0.7(0.1-3.6)$ & 0.0 & $2.3(0.4-8.1)$ \\
\hline
\end{tabular}

CI confidence interval, FMN first malignant neoplasm, SMN subsequent malignant neoplasm

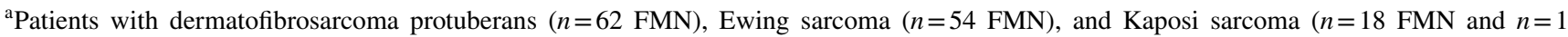
SMN) were excluded

${ }^{\mathrm{b}}$ Metastatic breast cancers (stage IV; $n=3$ breast-SMN patients and $n=22$ breast-FMN patients) and breast cancers with unknown stage ( $n=1$ breast-SMN patients and $n=2$ breast-FMN patients) were excluded

${ }^{c} 1$ control was excluded, because this patient appeared to have had a prior malignancy

${ }^{\mathrm{d}}$ Because of missing causes of death, the values do not always correspond with the overall survival numbers

${ }^{\mathrm{e}}$ Mortality due to other causes was considered a competing risk

${ }^{\mathrm{f}}$ Mortality due to the cancer of interest was considered a competing risk

a

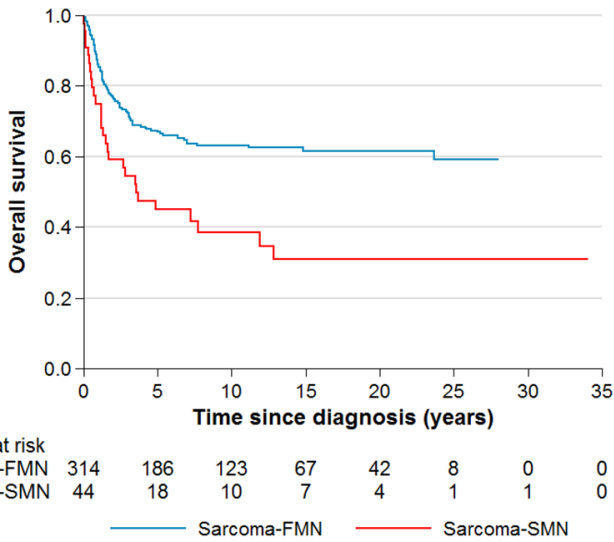

Fig. 1 Overall survival of patients with a subsequent malignant neoplasm $(\mathrm{SMN})$ and matched patients with a first malignant neoplasm (FMN) for sarcoma (a) and non-metastatic breast cancer (stage I-III) (b). Patients b

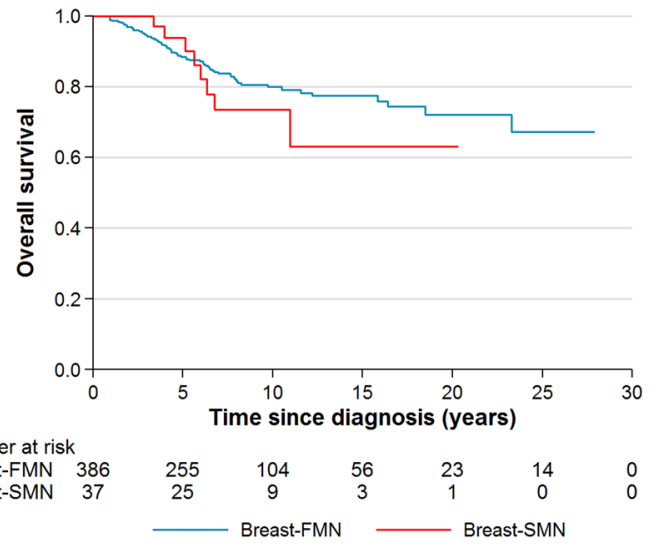

with dermatofibrosarcoma protuberans ( $n=62 \mathrm{FMN})$, Ewing sarcoma $(n=54 \mathrm{FMN})$, and Kaposi sarcoma $(n=18 \mathrm{FMN}$ and $n=1 \mathrm{SMN})$ were excluded in the sarcoma plot because adjuvant radiotherapy was impossible due to previous chest radiotherapy treatment for Hodgkin lymphoma and two breast-SMN patients had an indication for adjuvant chemotherapy, but were not treated because the maximum cumulative anthracycline dose would be exceeded.

\section{Breast cancer-overall and cause-specific survival}

Median follow-up time was 6.3 years (range $0.5-20.3$ years) for breast-SMN patients and 7.1 years (range 0.3-27.9 years) for breast-FMN patients. At end of 
Table 3 Regression analyses to ascertain the contribution of tumor and treatment characteristics between sarcoma as a subsequent malignant neoplasm (SMN) and sarcoma as a first malignant neoplasm (FMN) with respect to overall and cause-specific mortality

\begin{tabular}{|c|c|c|c|c|c|c|}
\hline & \multicolumn{2}{|c|}{ Overall mortality $^{\mathrm{a}}$} & \multicolumn{2}{|c|}{ Sarcoma-specific mortality ${ }^{\mathrm{b}}$} & \multicolumn{2}{|c|}{ Mortality other causes ${ }^{\mathrm{b}}$} \\
\hline & HR & $95 \% \mathrm{CI}$ & HR & $95 \% \mathrm{CI}$ & HR & $95 \% \mathrm{CI}$ \\
\hline Univariable SMN versus FMN & 2.13 & $1.41-3.22$ & 2.21 & $1.45-3.37$ & 1.13 & $0.14-9.74$ \\
\hline \multicolumn{7}{|l|}{ Multivariable } \\
\hline \multicolumn{7}{|l|}{ Model 1: adjustment tumor characteristics ${ }^{\mathrm{c}}$} \\
\hline SMN versus FMN & 1.83 & $1.21-2.78$ & 1.90 & $1.22-2.96$ & 1.06 & $0.13-8.47$ \\
\hline \multicolumn{7}{|l|}{ Morphology } \\
\hline Osteosarcoma/rhabdomyosarcoma & 1.00 (ref) & & 1.00 (ref) & & 1.00 (ref) & \\
\hline Chondrosarcoma & 0.12 & $0.05-0.29$ & 0.08 & $0.03-0.26$ & 0.44 & $0.05-3.96$ \\
\hline Other or unspecified & 0.66 & $0.47-0.92$ & 0.71 & $0.49-1.01$ & 0.26 & $0.05-1.38$ \\
\hline \multicolumn{7}{|c|}{ Model 2: adjustment treatment characteristics ${ }^{\mathrm{d}}$} \\
\hline SMN versus FMN & 1.90 & $1.25-2.90$ & 1.93 & $1.18-3.18$ & 0.98 & $0.07-14.54$ \\
\hline Surgery (yes vs. no) & $2.40^{\mathrm{e}}$ & $0.44-13.05^{\mathrm{e}}$ & 0.28 & $0.18-0.45$ & 0.38 & $0.03-5.44$ \\
\hline Radiotherapy (yes vs. no) & 1.44 & $1.01-2.06$ & 1.66 & $1.13-2.44$ & 0.47 & $0.05-4.23$ \\
\hline Chemotherapy (yes vs. no) & 1.88 & $1.29-2.73$ & 2.12 & $1.38-3.27$ & 0.44 & $0.04-5.49$ \\
\hline \multicolumn{7}{|c|}{ Model 3: adjustment tumor + treatment characteristics ${ }^{\mathrm{f}}$} \\
\hline SMN versus FMN & 1.88 & $1.23-2.87$ & 1.91 & $1.16-3.13$ & 0.77 & $0.05-10.97$ \\
\hline Surgery (yes vs. no) & $2.67^{\mathrm{g}}$ & $0.49-14.61^{\mathrm{g}}$ & 0.30 & $0.19-0.48$ & 0.47 & $0.04-4.98$ \\
\hline Radiotherapy (yes vs. no) & 1.29 & $0.90-1.85$ & 1.46 & $1.00-2.13$ & 0.58 & $0.06-5.18$ \\
\hline Chemotherapy (yes vs. no) & 1.68 & $1.07-2.63$ & 2.13 & $1.29-3.52$ & 0.13 & $0.02-0.97$ \\
\hline \multicolumn{7}{|l|}{ Morphology } \\
\hline Osteosarcoma/rhabdomyosarcoma & $1.00(\mathrm{ref})$ & & 1.00 (ref) & & 1.00 (ref) & \\
\hline Chondrosarcoma & 0.26 & $0.10-0.69$ & 0.23 & $0.07-0.77$ & 0.14 & $0.01-1.26$ \\
\hline Other or unspecified & 1.08 & $0.72-1.64$ & 1.33 & $0.85-2.06$ & 0.10 & $0.02-0.60$ \\
\hline
\end{tabular}

Patients with dermatofibrosarcoma protuberans ( $n=62 \mathrm{FMN})$, Ewing sarcoma $(n=54 \mathrm{FMN})$, and Kaposi sarcoma $(n=18 \mathrm{FMN}$ and $n=1 \mathrm{SMN})$ were excluded

CI confidence interval, FMN first malignant neoplasm, $H R$ hazard ratio, SMN subsequent malignant neoplasm

${ }^{a}$ Cox proportional hazards regression analyses including 28 deaths among sarcoma-SMN patients and 114 deaths among sarcoma-FMN patients

${ }^{\mathrm{b}}$ Competing risk regression analyses. When evaluating sarcoma-specific mortality, mortality due to other causes was considered a competing risk and vice versa

${ }^{c}$ Adjusted for morphology (osteosarcoma/Ewing sarcoma/rhabdomyosarcoma; chondrosarcoma; other or unspecified)

${ }^{\mathrm{d}}$ Adjusted for surgery (yes vs. no), radiotherapy (yes vs. no), and chemotherapy (yes vs. no)

eProportional hazards assumption was violated for category "Surgery (yes vs. no)." At a follow-up duration of 6.41 years (median), the hazard ratio was 0.99 (95\% CI: $0.40-2.44$ ) with an increase of a factor $1.58^{\wedge}$ (years of follow-up after 6.41 years)

${ }^{\mathrm{f}}$ Adjusted for morphology, surgery, radiotherapy, and chemotherapy

gProportional hazards assumption was violated for category "Surgery (yes vs. no)." At a follow-up duration of 6.41 years (median), the hazard ratio was 2.67 (95\% CI 0.49-14.61) with an increase of a factor $1.59^{\wedge}$ (years of follow-up after 6.41 years)

follow-up, 12 (29.3\%) breast-SMN patients were deceased compared to $89(21.7 \%)$ breast-FMN patients (Table 4$)$. For non-metastatic breast cancer (stage I-III), overall survival rates after 5 and 15 years were $93.8 \%(95 \%$ CI $77.4-98.4 \%)$ and $63.1 \%$ (95\% CI 34.9-81.8\%) among breast-SMN patients and $88.5 \%$ (95\% CI $84.7-91.4 \%)$ and 77.4\% (95\% CI 71.5-82.2\%) among breast-FMN patients, respectively (Table 2, Fig. 1b). Fifteen-year survival rates among all patients with breast cancer (including metastatic) were $47.6 \%$ (95\% CI 21.9-69.5\%) for breast-SMN and $73.2 \%$ (95\% CI 67.3-78.1\%) for breast-FMN patients.
For non-metastatic breast cancer, breast cancer-specific mortality at 15 -year was $33.2 \%$ (95\% CI $12.2-56.1 \%$ ) for breast-SMN patients and $19.8 \%$ (95\% CI 15.0-25.1\%) for breast-FMN patients (Table 2). Mortality due to other causes was very low $(0-4 \%)$. Overall survival did not significantly differ between breast-SMN patients and breastFMN patients (multivariable HR 1.14; 95\% CI 0.54-2.37) (Table 5). Similar trends were observed for breast cancer-specific mortality (multivariable HR 1.09; 95\% CI $0.49-2.39)$. 
Table 4 Characteristics of patients with female breast cancer as a subsequent malignant neoplasm (SMN) and matched patients with female breast cancer as a firs malignant neoplasm (FMN)

\begin{tabular}{|c|c|c|c|c|c|}
\hline & \multicolumn{2}{|c|}{$\begin{array}{l}\text { DCOG-LATER } \\
\text { cohort SMN } \\
(n=41)\end{array}$} & \multicolumn{2}{|c|}{$\begin{array}{l}\text { Cancer registry } \\
\text { FMN } \\
(n=410)\end{array}$} & \multirow[t]{2}{*}{$p$ value } \\
\hline & $N$ & $\%$ & $N$ & $\%$ & \\
\hline Age at diagnosis ${ }^{\mathrm{a}}$ & & & & & 1.000 \\
\hline$<30$ years & 6 & 14.6 & 60 & 14.6 & \\
\hline $30-39$ years & 23 & 56.1 & 230 & 56.1 & \\
\hline $40-49$ years & 8 & 19.5 & 80 & 19.5 & \\
\hline $50+$ years & 4 & 9.8 & 40 & 9.8 & \\
\hline Incidence year $^{\mathrm{a}}$ & & & & & 1.000 \\
\hline 1989-1999 & 7 & 17.1 & 70 & 17.1 & \\
\hline 2000-2009 & 20 & 48.8 & 200 & 48.8 & \\
\hline 2010-2014 & 14 & 34.2 & 140 & 34.2 & \\
\hline Morphology breast cancer & & & & & 0.379 \\
\hline Ductal & 36 & 87.8 & 358 & 87.3 & \\
\hline Lobular & 3 & 7.3 & 21 & 5.1 & \\
\hline Mixed ductal/lobular & 2 & 4.9 & 12 & 2.9 & \\
\hline Other $^{b}$ & 0 & 0.0 & 19 & 4.6 & \\
\hline Stage $^{c}$ & & & & & 0.499 \\
\hline I & 16 & 40.0 & 126 & 30.9 & \\
\hline II & 15 & 37.5 & 197 & 48.3 & \\
\hline III & 6 & 15.0 & 63 & 15.4 & \\
\hline IV & 3 & 7.5 & 22 & 5.4 & \\
\hline Unknown & 1 & & 2 & & \\
\hline Grade $^{c}$ & & & & & 0.172 \\
\hline Well differentiated & 2 & 6.9 & 37 & 12.2 & \\
\hline Moderately differentiated & 15 & 51.7 & 103 & 33.9 & \\
\hline Poorly differentiated & 12 & 41.4 & 164 & 54.0 & \\
\hline Unknown & 12 & & 106 & & \\
\hline ER status $^{c}$ & & & & & 0.202 \\
\hline Negative & 5 & 16.1 & 83 & 28.1 & \\
\hline Positive & 26 & 83.9 & 212 & 71.9 & \\
\hline Unknown & 10 & & 115 & & \\
\hline PR status $^{\mathrm{c}}$ & & & & & 0.315 \\
\hline Negative & 8 & 28.6 & 114 & 38.9 & \\
\hline Positive & 20 & 71.4 & 179 & 61.1 & \\
\hline Unknown & 13 & & 117 & & \\
\hline Her2 receptor status ${ }^{c, e}$ & & & & & 0.803 \\
\hline Negative & 20 & 76.9 & 210 & 79.0 & \\
\hline Positive & 6 & 23.1 & 56 & 21.1 & \\
\hline Unknown & 15 & & 144 & & \\
\hline Receptor status ${ }^{\mathrm{c}}$ & & & & & 0.393 \\
\hline Triple negative & 2 & 7.7 & 55 & 20.8 & \\
\hline ER and PR negative, Her2 receptor positive & 1 & 3.9 & 14 & 5.3 & \\
\hline ER and/or PR positive, Her2 receptor positive & 5 & 19.2 & 40 & 15.2 & \\
\hline ER and/or PR positive, Her2 receptor negative & 18 & 69.2 & 155 & 58.7 & \\
\hline Unknown & 15 & & 146 & & \\
\hline Surgery $^{c}$ & & & & & 0.024 \\
\hline No & 4 & 9.8 & 13 & 3.2 & \\
\hline Mastectomy & 26 & 63.4 & 202 & 49.3 & \\
\hline Breast-conserving surgery & 11 & 26.8 & 193 & 47.1 & \\
\hline Surgery, type unknown & 0 & 0.0 & 2 & 0.5 & \\
\hline
\end{tabular}


Table 4 (continued)

\begin{tabular}{|c|c|c|c|c|c|}
\hline & \multicolumn{2}{|c|}{$\begin{array}{l}\text { DCOG-LATER } \\
\text { cohort SMN } \\
(n=41)\end{array}$} & \multicolumn{2}{|c|}{$\begin{array}{l}\text { Cancer registry } \\
\text { FMN } \\
(n=410)\end{array}$} & \multirow[t]{2}{*}{$p$ value } \\
\hline & $N$ & $\%$ & $N$ & $\%$ & \\
\hline Radiotherapy & & & & & 0.011 \\
\hline No & 20 & 48.8 & 116 & 28.3 & \\
\hline Yes & 21 & 51.2 & 294 & 71.7 & \\
\hline Chemotherapy & & & & & 0.022 \\
\hline No & 17 & 41.5 & 97 & 23.7 & \\
\hline Yes & 24 & 58.5 & 313 & 76.3 & \\
\hline Hormonal therapy & & & & & 0.870 \\
\hline No & 23 & 56.1 & 219 & 53.4 & \\
\hline Yes & 18 & 43.9 & 191 & 46.6 & \\
\hline Treatment category & & & & & 0.011 \\
\hline No surgery ${ }^{\mathrm{d}}$ & 4 & 9.8 & 13 & 3.2 & \\
\hline Mastectomy, no RT or CT $( \pm \mathrm{HT})$ & 7 & 17.1 & 23 & 5.6 & \\
\hline Mastectomy + RT and/or CT $( \pm \mathrm{HT})$ & 19 & 46.3 & 179 & 43.7 & \\
\hline Breast-conserving surgery, no RT or CT $( \pm \mathrm{HT})$ & 0 & 0.0 & 2 & 0.5 & \\
\hline Breast-conserving surgery $+\mathrm{RT}$ and/or $\mathrm{CT}( \pm \mathrm{HT})$ & 11 & 26.8 & 191 & 46.6 & \\
\hline Surgery, type unknown & 0 & 0.0 & 2 & 0.5 & \\
\hline Vital status at end of follow-up & & & & & 0.325 \\
\hline Alive & 29 & 70.7 & 321 & 78.3 & \\
\hline Deceased & 12 & 29.3 & 89 & 21.7 & \\
\hline
\end{tabular}

$C T$ chemotherapy, ER estrogen receptor, $F M N$ first malignant neoplasm, $H T$ hormonal therapy, $P R$ progesterone receptor, $R T$ radiotherapy, $S M N$ subsequent malignant neoplasm

${ }^{\text {a }}$ Matching factors

${ }^{b}$ Includes seven medullary carcinomas, six mucinous adenocarcinomas, two tubular adenocarcinomas, one each of small cell neuroendocrine carcinoma, apocrine adenocarcinoma, medullary carcinoma with lymphoid stroma, and metaplastic carcinoma

${ }^{c}$ Percentages and $p$ values are based on subjects with known information only

${ }^{\mathrm{d}}$ Among breast-SMN cases, two were treated with CT only and 1 each with HT only and no recorded treatment. Among breast-FMN cases, five were treated with CT only, two each with RT $+\mathrm{CT}, \mathrm{CT}+\mathrm{HT}$, and no recorded treatment, and one each with $\mathrm{RT}+\mathrm{HT}$ and HT only

${ }^{\mathrm{e}} \mathrm{Her} 2$ receptor status has been systematically ascertained from September 2005 and patients with a positive Her2 receptor status have been treated with Herceptin according to Dutch treatment guidelines since then

\section{Melanoma-comparison of clinical and histopathological characteristics}

Of 18 eligible patients with subsequent melanoma (melanoma-SMN) after childhood cancer, one patient was excluded because of a history of radiotherapy and chemotherapy for an intervening ALL diagnosis. We included 17 melanoma-SMN patients and 170 patients with first primary melanoma (melanoma-FMN). More than forty percent of melanoma-SMN cases $(41.2 \%)$ occurred after childhood leukemia and $23.5 \%$ of melanoma-SMN cases had prior radiotherapy to the melanoma region (Supplementary Table 2).

Among patients with melanoma-SMN, $88.2 \%$ were diagnosed with superficial spreading melanoma, compared to $68.8 \%$ among melanoma-FMN patients. No significant differences between melanoma-SMN and melanoma-FMN patients were observed for stage distribution and melanoma treatment (Table 1).

\section{Melanoma-overall and cause-specific survival}

Median follow-up time was 9.4 years (range 0.9-20.9 years) for melanoma-SMN patients and 9.2 years (range 0.4-20.9 years) for melanoma-FMN patients. At end of follow-up, 11.8\% of melanoma-SMN patients were deceased compared to $10.0 \%$ of melanoma-FMN patients (Table 1). Fifteen-year survival exceeded $80 \%$ in both patients with melanoma-SMN (82\%) and melanoma-FMN (87\%) (Table 2). Melanoma-specific mortality at 15 year since diagnosis was $12.5 \%$ for melanomaSMN and $10.0 \%$ for melanoma-FMN patients. No significant differences were observed between melanoma-SMN and melanoma-FMN patients in overall survival (multivariable 
Table 5 Regression analyses to ascertain the contribution of tumor and treatment characteristics between female breast cancer as a subsequent malignant neoplasm (SMN) and female breast cancer as a first malignant neoplasm (FMN) with respect to overall and cause-specific mortality

\begin{tabular}{|c|c|c|c|c|}
\hline & \multicolumn{2}{|c|}{$\begin{array}{l}\text { Overall } \\
\text { mortality }^{\mathrm{a}}\end{array}$} & \multicolumn{2}{|c|}{$\begin{array}{l}\text { Breast cancer- } \\
\text { specific } \\
\text { mortality }\end{array}$} \\
\hline & HR & $95 \% \mathrm{CI}$ & HR & $95 \% \mathrm{CI}$ \\
\hline Univariable SMN versus FMN & 1.45 & $0.79-2.65$ & 1.43 & $0.79-2.59$ \\
\hline \multicolumn{5}{|l|}{ Multivariable } \\
\hline \multicolumn{5}{|c|}{ Model 1: adjustment tumor characteristic ${ }^{c}$} \\
\hline SMN versus FMN & 1.32 & $0.70-2.47$ & 1.30 & $0.65-2.59$ \\
\hline Stage (III/IV vs. I/II) & 4.96 & $3.30-7.45$ & 5.55 & $3.62-8.53$ \\
\hline \multicolumn{5}{|c|}{ Model 2: adjustment treatment characteristics ${ }^{\mathrm{d}}$} \\
\hline SMN versus FMN & 1.23 & $0.61-2.47$ & 1.19 & $0.61-2.32$ \\
\hline $\begin{array}{l}\text { Surgery type (mastectomy vs. } \\
\text { breast-conserving or type } \\
\text { unknown) }\end{array}$ & 3.15 & $1.86-5.34$ & 3.45 & $1.85-6.41$ \\
\hline Radiotherapy (yes vs. no) & 2.14 & $1.23-3.72$ & 2.35 & $1.24-4.47$ \\
\hline Chemotherapy (yes vs. no) & 0.79 & $0.47-1.35$ & 0.86 & $0.47-1.56$ \\
\hline \multicolumn{5}{|c|}{ Model 3: adjustment tumor + treatment characteristics ${ }^{\mathrm{e}}$} \\
\hline SMN versus FMN & 1.14 & $0.54-2.37$ & 1.09 & $0.49-2.39$ \\
\hline Stage (III/IV vs. I/II) & 2.92 & $1.62-5.26$ & 3.09 & $1.39-6.85$ \\
\hline $\begin{array}{l}\text { Surgery type (mastectomy vs. } \\
\text { breast-conserving or type } \\
\text { unknown) }\end{array}$ & 1.90 & $1.02-3.53$ & 2.03 & $0.87-4.78$ \\
\hline Radiotherapy (yes vs. no) & 1.30 & $0.66-2.53$ & 1.40 & $0.54-3.62$ \\
\hline Chemotherapy (yes vs. no) & 0.77 & $0.44-1.33$ & 0.84 & $0.44-1.60$ \\
\hline
\end{tabular}

$C I$ confidence interval, FMN first malignant neoplasm, $H R$ hazard ratio, $S M N$ subsequent malignant neoplasm

${ }^{a}$ Cox proportional hazards regression analyses including 12 deaths among breast-SMN patients and 89 deaths among breast-FMN patients

${ }^{\mathrm{b}}$ Competing risk regression analyses. Mortality due to other causes was considered a competing risk and vice versa. Univariable HR for mortality due to other causes: 10.00 (95\% CI 0.64-156.62)

${ }^{\mathrm{c}}$ Adjusted for stage (III/IV vs. I/II). One breast-SMN patient and two breast-FMN patients with unknown stage were excluded

${ }^{\mathrm{d}}$ Adjusted for surgery type (mastectomy vs. breast-conserving or type unknown), radiotherapy (yes vs. no), and chemotherapy (yes vs. no). One breast-SMN patient and two breast-FMN patients without surgery were excluded

${ }^{\mathrm{e}}$ Adjusted for stage, surgery type, radiotherapy, and chemotherapy. Two breast-SMN patients and four first-SMN patients were excluded because of missing stage or no surgical treatment

HR 0.80; 95\% CI 0.10-6.21) and melanoma-specific survival (univariable HR 0.71; 95\% CI 0.10-5.00) (Supplementary Table 4).

\section{Discussion}

In this study, survival and characteristics of three types of solid SMNs from a large cohort of childhood cancer survivors were compared to FMNs of the same type in the general population. Survival of patients with sarcomas after childhood cancer was poor and significantly worse than survival of patients with sarcomas as FMNs. Although we observed that breast-SMN patients more often received a mastectomy without radiotherapy or chemotherapy compared to breast-FMN patients, survival was not different between breast-SMN and breast-FMN patients.

This is one of the few studies to compare SMNs among childhood cancer survivors with FMNs occurring in an age- and diagnosis-era matched population-based sample of cancer patients. We found that overall survival was worse for sarcoma-SMN patients than for sarcoma-FMN patients, both for bone sarcoma and soft-tissue sarcoma. This worse survival is in line with results from two registry-based studies in the United States comparing sarcomaSMN with sarcoma-FMN patients across patients of all ages $[14,23]$ and with two studies comparing sarcomaSMN patients with prior irradiation to sarcoma-FMN patients [24, 25]. We observed a considerable shift in type of sarcoma seen after childhood cancer as SMN vs. those seen as FMN, in particular a shift from Ewing sarcoma and dermatofibrosarcoma in the FMN group to osteosarcoma in the SMN group. The group of sarcoma-SMN patients may harbor more patients with a genetic predisposition than sarcoma-FMN patients, especially those patients with sarcoma-SMNs occurring after retinoblastoma or Li-Fraumeni syndrome-associated tumors [26-28]. The stage distribution was not different between the groups in our study. Robison et al. found that sarcoma-SMN patients who had received prior radiotherapy to the sarcoma region seemed to have a more advanced stage than sarcoma-FMN patients, although this difference was not statistically significant [23]. Similar to what Robison et al. reported, grade seemed to be worse among sarcoma-SMN patients in our study. However, in both studies, the majority of grades were unknown, so no firm conclusions can be drawn [23]. Bjerkehagen et al. showed that the poorer prognosis of sarcomas occurring in previous radiation fields compared to sporadic sarcomas could be explained by more unfavorable factors, such as central tumor site and incomplete surgical remission [24]. Treatment modalities administered for sarcoma treatment did not differ between sarcoma-SMN and sarcoma-FMN patients in our study. Although this may indicate that treatment generally was not influenced by previous childhood cancer treatment, a note of caution is warranted since administration of specific, effective agents (in particular anthracyclines) may 
differ between the groups. This level of detail is not available for the cancer registry sample of FMN patients. Also, the differences with respect to sarcoma subtypes between the groups have somewhat limited the clinical impact of our comparisons.

Breast-SMN and breast-FMN patients generally had comparable tumor characteristics. No differences between the two groups were observed with regard to stage and grade distribution, in accordance with two small studies among HL survivors [13, 29]. In contrast to our findings, two large registry-based studies in AYA cancer survivors did report that AYA cancer/HL survivors with breast SMNs were significantly more likely to have estrogen- and progestogennegative breast cancer compared to breast-FMN patients [16, 17]. The percentage of triple negative breast cancer in our study was lower than that reported in a retrospective case series of breast cancer patients with a past history of chest radiotherapy (7.7\% vs. 29.2\%) [30]. An important observation in our study was the higher proportion of breast-SMN patients who had received a mastectomy compared to breastFMN patients, in particular the proportion of mastectomy without radiotherapy or chemotherapy (17\% vs. $6 \%)$, as reported earlier [10, 13, 16, 17, 29, 31]. Prior chest radiotherapy treatment for childhood cancer may have limited the adjuvant treatment options after breast-conserving surgery and therefore mastectomy may have been the treatment of choice. In one breast-SMN patient with a mastectomy without radiotherapy or chemotherapy, we found evidence that indeed prior childhood cancer radiotherapy led to the choice for mastectomy over breast-conserving surgery. We found no significant difference in overall survival between breast-SMN and breast-FMN patients. However, patients with breast-SMN seemed to have slightly higher late cumulative mortality rates (10-year and 15-year) than breast-FMN patients, although these differences were not statistically significant. Although the high percentage of breast cancer as the cause of death among deceased patients is suggestive of breast-SMN patients experiencing more often late recurrences/metastases than breast-FMN patients, numbers were too small to interpret. Previous registry-based followup studies in Hodgkin lymphoma survivors or in childhood and young adult cancer survivors did find a significantly increased mortality risk in breast-SMN patients compared to breast-FMN patients $[14,16,17,29,31]$. Three smaller single-institution studies among (Hodgkin) lymphoma survivors showed mixed results, with one also showing significantly worse survival in breast-SMN patients [18], while the other study did not find a difference between breast-SMN and breast-FMN patients $[10,13]$. We observed that the large majority of breast-SMN patients died because of the breast cancer, in contrast to Milano et al. who found that many of the breast-SMN patients after Hodgkin lymphoma died of other cancers, heart disease, or other causes [16].
The high risks of other fatal late adverse effects among patients with Hodgkin lymphoma treated with high dose, large volume radiotherapy, and/or high doses of chemotherapy [32] can probably not be extrapolated to that in a mixed cohort of childhood cancer survivors with a large proportion of breast cancers in non-irradiated survivors. Compared to Hodgkin lymphoma survivors, the group of childhood cancer survivors harbors a subpopulation of individuals with TP53-related or other genetic predisposition profiles that predispose to breast cancer. Therefore, it is possible that the related breast tumors are biologically different as well, although this a mere hypothesis at this point. Because breast cancers in the breast-SMN group were diagnosed at fairly young ages, and thus matched breast-FMN group represents a sample of young patients, it is likely that the breast-FMN group includes several women with a genetic predisposition, such as BRCA mutation carriers. Overall survival among young individuals with melanoma is very good, with few fatalities among SMN and FMN cases alike. MelanomaSMN patients harbor no worse prognosis profile vs. melanoma-FMN patients and there are no strong predictors of mortality. Keegan et al. did find a slight significantly worse overall survival in melanoma-SMN compared to melanomaFMN [14]. Previous studies have shown that childhood cancer survivors are at increased risk of skin cancers, mainly basal cell carcinomas, occurring mainly in skin surface areas that had received radiation [33, 34]. Survivors who received radiotherapy should be educated to be on alert for local skin changes in order to timely seek medical attention for skin lesions that may be potentially malignant.

Strengths of our study are the availability of clinical, histopathological, and treatment data of solid SMNs from a large, nationwide cohort of childhood cancer survivors and a matched population-based comparison group of FMNs. Furthermore, we were able to not only compare survival, but also causes of death, which enabled us to evaluate whether survival differences were caused by differences in cancerspecific mortality or mortality from other causes. A limitation was the relatively small numbers of site-specific SMNs included in our study, which may have limited the power to detect differences in clinical characteristics and survival between the cancer groups. Furthermore, for several tumor characteristics (e.g., sarcoma stage and grade and breast cancer receptor status information), there was a relatively high percentage of the data missing, in particular for cases diagnosed in the distant past, when cancer registration (and in fact clinical practice) did not include such detail. This omission limits impact of these findings.

In conclusion, our exploratory analyses reveal that survival of sarcoma-SMN patients is poor, with less than one in three surviving 15 years after sarcoma-SMN diagnosis, and is worse than survival of sarcoma-FMN patients. This observation seems partly related to differences in sarcoma 
subtypes. In our study, breast cancer survival and breast tumor characteristics are not different between breast-SMN and breast-FMN patients, but treatment differs as breastSMN patients receive more often a mastectomy than breastFMN patients. Survival of melanoma-SMN is favorable. Further studies including more cases should elaborate on possible explanations of a worse survival of (soft tissue) sarcoma-SMN patients and on treatment strategies and underlying deliberations among breast-SMN patients.

Acknowledgments We thank all data managers in the seven participating centers and Aslihan Mantici for obtaining the data for this study. Furthermore, we thank the following other members of the DCOGLATER group for their contributions: Dorine Bresters, Lilian Batenburg, Margreet Veening, Gea Huizinga, Lideke van der Steeg, Monique Jaspers, and Andrica de Vries. We thank the staff of the Netherlands Cancer Registry and Statistics Netherlands for providing data for this study.

The DCOG-LATER Study Group includes the listed authors and the following collaborators BMP Aleman (The Netherlands Cancer Institute, Amsterdam). HN Caron (Emma Children's Hospital/Academic Medical Center, Amsterdam). MA Grootenhuis (Emma Children's Hospital/Academic Medical Center, Amsterdam, and Princess Máxima Center for Pediatric Oncology, Utrecht). JG den Hartogh (Dutch Childhood Cancer Parent Organisation (VOKK), Nieuwegein). N Hollema (Dutch Childhood Oncology Group, Utrecht). SJCMM Neggers (Erasmus Medical Center, Rotterdam). A Postma (Dutch Childhood Oncology Group, The Hague). JG de Ridder-Sluiter (Princess Máxima Center for Pediatric Oncology, Utrecht). EJT Rutgers (The Netherlands Cancer Institute, Amsterdam).

Funding This study was funded by the Dutch Cancer Society (Grant Numbers DCOG2011-5027 and UVA2012-5517) and the European Union's Seventh Framework Programme for research, technological development, and demonstration under Grant Agreement No. 257505 (PanCareSurFup).

\section{Compliance with ethical standards}

Conflict of interest The authors declare that they have no conflict of interest.

Research involving human rights Data collection for the study was exempted from review by the Institutional Review Boards of all participating centers and all data were processed and analyzed anonymously.

Open Access This article is distributed under the terms of the Creative Commons Attribution 4.0 International License (http://creativeco mmons.org/licenses/by/4.0/), which permits unrestricted use, distribution, and reproduction in any medium, provided you give appropriate credit to the original author(s) and the source, provide a link to the Creative Commons license, and indicate if changes were made.

\section{References}

1. Bhakta N, Liu Q, Ness KK, Baassiri M, Eissa H, Yeo F, Chemaitilly W, Ehrhardt MJ, Bass J, Bishop MW, Shelton K, Lu L, Huang S, Li Z, Caron E, Lanctot J, Howell C, Folse T, Joshi V, Green DM, Mulrooney DA, Armstrong GT, Krull KR, Brinkman
TM, Khan RB, Srivastava DK, Hudson MM, Yasui Y, Robison LL (2017) The cumulative burden of surviving childhood cancer: an initial report from the St Jude Lifetime Cohort Study (SJLIFE). Lancet 390(10112):2569-2582

2. Geenen MM, Cardous-Ubbink MC, Kremer LC, van den Bos C, van der Pal HJ, Heinen RC, Jaspers MW, Koning CC, Oldenburger F, Langeveld NE, Hart AA, Bakker PJ, Caron HN, van Leeuwen FE (2007) Medical assessment of adverse health outcomes in long-term survivors of childhood cancer. JAMA 297(24):2705-2715

3. Hudson MM, Ness KK, Gurney JG, Mulrooney DA, Chemaitilly W, Krull KR, Green DM, Armstrong GT, Nottage KA, Jones KE, Sklar CA, Srivastava DK, Robison LL (2013) Clinical ascertainment of health outcomes among adults treated for childhood cancer. JAMA 309(22):2371-2381

4. Oeffinger KC, Mertens AC, Sklar CA, Kawashima T, Hudson MM, Meadows AT, Friedman DL, Marina N, Hobbie W, Kadan-Lottick NS, Schwartz CL, Leisenring W, Robison LL, Childhood Cancer Survivor S (2006) Chronic health conditions in adult survivors of childhood cancer. N Engl J Med 355(15):1572-1582

5. Armstrong GT, Liu Q, Yasui Y, Neglia JP, Leisenring W, Robison LL, Mertens AC (2009) Late mortality among 5-year survivors of childhood cancer: a summary from the Childhood Cancer Survivor Study. J Clin Oncol 27(14):2328-2338

6. Fidler MM, Reulen RC, Winter DL, Kelly J, Jenkinson HC, Skinner R, Frobisher C, Hawkins MM, British Childhood Cancer Survivor Study Steering G (2016) Long term cause specific mortality among 34489 five year survivors of childhood cancer in Great Britain: population based cohort study. BMJ 354:i4351

7. Reulen RC, Winter DL, Frobisher C, Lancashire ER, Stiller CA, Jenney ME, Skinner R, Stevens MC, Hawkins MM, British Childhood Cancer Survivor Study Steering G (2010) Long-term causespecific mortality among survivors of childhood cancer. JAMA 304(2):172-179

8. Teepen JC, van Leeuwen FE, Tissing WJ, van Dulmen-den Broeder E, van den Heuvel-Eibrink MM, van der Pal HJ, Loonen JJ, Bresters D, Versluys B, Neggers S, Jaspers MWM, Hauptmann M, Heiden-van der Loo M, Visser O, Kremer LCM, Ronckers CM (2017) Long-Term Risk of Subsequent Malignant Neoplasms After Treatment of Childhood Cancer in the DCOG LATER Study Cohort: Role of Chemotherapy. J Clin Oncol 35(20):2288-2298

9. Turcotte LM, Liu Q, Yasui Y, Arnold MA, Hammond S, Howell RM, Smith SA, Weathers RE, Henderson TO, Gibson TM, Leisenring W, Armstrong GT, Robison LL, Neglia JP (2017) Temporal trends in treatment and subsequent neoplasm risk among 5-year survivors of childhood cancer, 1970-2015. JAMA 317(8):814-824

10. Alm El-Din MA, Hughes KS, Raad RA, Goldberg SI, Aisenberg AC, Niemierko A, Taghian AG (2009) Clinical outcome of breast cancer occurring after treatment for Hodgkin's lymphoma: casecontrol analysis. Radiat Oncol 4:19

11. Chowdhry AK, Fung C, Chowdhry VK, Bergsma D, Dhakal S, Constine LS, Milano MT (2018) A population-based study of prognosis and survival in patients with second primary thyroid cancer after Hodgkin lymphoma. Leuk Lymphoma 59(5):1180-1187

12. Goldfarb M, Freyer DR (2014) Comparison of secondary and primary thyroid cancer in adolescents and young adults. Cancer 120(8):1155-1161

13. Janov AJ, Tulecke M, O'Neill A, Lester S, Mauch PM, Harris J, Schnitt SJ, Shapiro CL (2001) Clinical and pathologic features of breast cancers in women treated for Hodgkin's disease: a casecontrol study. Breast J 7(1):46-52 
14. Keegan THM, Bleyer A, Rosenberg AS, Li Q, Goldfarb M (2017) Second primary malignant neoplasms and survival in adolescent and young adult cancer survivors. JAMA Oncol 3(11):1554-1557

15. Milano MT, Li H, Constine LS, Travis LB (2011) Survival after second primary lung cancer: a population-based study of 187 Hodgkin lymphoma patients. Cancer 117(24):5538-5547

16. Milano MT, Li H, Gail MH, Constine LS, Travis LB (2010) Longterm survival among patients with Hodgkin's lymphoma who developed breast cancer: a population-based study. J Clin Oncol 28(34):5088-5096

17. Sadler C, Goldfarb M (2015) Comparison of primary and secondary breast cancers in adolescents and young adults. Cancer 121(8):1295-1302

18. Sanna G, Lorizzo K, Rotmensz N, Bagnardi V, Cinieri S, Colleoni M, Nole F, Goldhirsch A (2007) Breast cancer in Hodgkin's disease and non-Hodgkin's lymphoma survivors. Ann Oncol 18(2):288-292

19. Youn P, Li H, Milano MT, Stovall M, Constine LS, Travis LB (2013) Long-term survival among Hodgkin's lymphoma patients with gastrointestinal cancer: a population-based study. Ann Oncol 24(1):202-208

20. Forman D, Bray F, Brewster DH, Gombe Mbalawa C, Kohler B, Piñeros M, Steliarova-Foucher E, Swaminathan R, Ferlay J (2014) Cancer incidence in five continents. Vol. X. IARC Scientific Publications No. 164. Lyon: International Agency for Research on Cancer

21. Fine JP, Gray RJ (1999) A proportional hazards model for the subdistribution of a competing risk. J Am Stat Assoc 94(446):496-509

22. Criscione VD, Weinstock MA (2007) Descriptive epidemiology of dermatofibrosarcoma protuberans in the United States, 1973 to 2002. J Am Acad Dermatol 56(6):968-973

23. Robinson E, Bar-Deroma R, Rennert G, Neugut AI (1992) A comparison of the clinical characteristics of second primary and single primary sarcoma: a population based study. J Surg Oncol 50(4):263-266

24. Bjerkehagen B, Smastuen MC, Hall KS, Skjeldal S, Smeland S, Fossa SD (2012) Why do patients with radiation-induced sarcomas have a poor sarcoma-related survival? Br J Cancer 106(2):297-306

25. Gladdy RA, Qin LX, Moraco N, Edgar MA, Antonescu CR, Alektiar KM, Brennan MF, Singer S (2010) Do radiation-associated soft tissue sarcomas have the same prognosis as sporadic soft tissue sarcomas? J Clin Oncol 28(12):2064-2069

26. Bright CJ, Hawkins MM, Winter DL, Alessi D, Allodji RS, Bagnasco F, Bardi E, Bautz A, Byrne J, Feijen EAM, Fidler MM, Garwicz S, Grabow D, Gudmundsdottir T, Guha J, Haddy N, Jankovic M, Kaatsch P, Kaiser M, Kuehni CE, Linge H, Ofstaas H, Ronckers CM, Skinner R, Teepen JC, Terenziani M, Vu-Bezin
G, Wesenberg F, Wiebe T, Sacerdote C, Jakab Z, Haupt R, Lahteenmaki P, Zaletel LZ, Kuonen R, Winther JF, de Vathaire F, Kremer LC, Hjorth L, Reulen RC, PanCareSurFup C (2018) Risk of Soft-Tissue Sarcoma Among 69460 Five-Year Survivors of Childhood Cancer in Europe. J Natl Cancer Inst 110(6):649-660

27. Kleinerman RA, Tucker MA, Abramson DH, Seddon JM, Tarone RE, Fraumeni JF Jr (2007) Risk of soft tissue sarcomas by individual subtype in survivors of hereditary retinoblastoma. J Natl Cancer Inst 99(1):24-31

28. Li FP, Fraumeni JF Jr (1969) Soft-tissue sarcomas, breast cancer, and other neoplasms. A familial syndrome? Ann Intern Med 71(4):747-752

29. Veit-Rubin N, Rapiti E, Usel M, Benhamou S, Vinh-Hung V, Vlastos G, Bouchardy C (2012) Risk, characteristics, and prognosis of breast cancer after Hodgkin's lymphoma. Oncologist 17(6):783-791

30. Demoor-Goldschmidt C, Supiot S, Mahe MA, Oberlin O, Allodji R, Haddy N, Helfre S, Vigneron C, Brillaud-Meflah V, Bernier V, Laprie A, Ducassou A, Claude L, Diallo I, de Vathaire F (2018) Clinical and histological features of second breast cancers following radiotherapy for childhood and young adult malignancy. $\mathrm{Br} \mathbf{J}$ Radiol 91(1086):20170824

31. Elkin EB, Klem ML, Gonzales AM, Ishill NM, Hodgson D, Ng AK, Marks LB, Weidhaas J, Freedman GM, Miller RC, Constine LS, Myrehaug S, Yahalom J (2011) Characteristics and outcomes of breast cancer in women with and without a history of radiation for Hodgkin's lymphoma: a multi-institutional, matched cohort study. J Clin Oncol 29(18):2466-2473

32. van Leeuwen FE, Ng AK (2017) Late sequelae in Hodgkin lymphoma survivors. Hematol Oncol 35(Suppl 1):60-66

33. Perkins JL, Liu Y, Mitby PA, Neglia JP, Hammond S, Stovall M, Meadows AT, Hutchinson R, Dreyer ZE, Robison LL, Mertens AC (2005) Nonmelanoma skin cancer in survivors of childhood and adolescent cancer: a report from the childhood cancer survivor study. J Clin Oncol 23(16):3733-3741

34. Teepen JC, Kok JL, Kremer LC, Tissing WJE, van den HeuvelEibrink MM, Loonen JJ, Bresters D, van der Pal HJ, Versluys B, van Dulmen-den Broeder E, Nijsten T, Hauptmann M, Hollema N, Dolsma WV, van Leeuwen FE, Ronckers CM, Group D-LS (2019) Long-term risk of skin cancer among childhood cancer survivors: A DCOG-LATER Cohort Study. J Natl Cancer Inst. https://doi. org/10.1093/jnci/djy212

Publisher's Note Springer Nature remains neutral with regard to jurisdictional claims in published maps and institutional affiliations.

\section{Affiliations}

\section{Jop C. Teepen ${ }^{1,2}\left(\mathbb{D} \cdot\right.$ Leontien C. Kremer ${ }^{1,2} \cdot$ Margriet van der Heiden-van der Loo ${ }^{3}$. Wim J. Tissing ${ }^{2,4}$. Helena J. van der Pal ${ }^{2} \cdot$ Marry M. van den Heuvel-Eibrink ${ }^{2,5}$. Jacqueline J. Loonen ${ }^{6} \cdot$ Marloes Louwerens $^{7}$. Birgitta Versluys $^{8} \cdot$ Eline van Dulmen-den Broeder $^{9} \cdot$ Otto Visser $^{10}$. John H. Maduro ${ }^{11}$. Flora E. van Leeuwen ${ }^{12}$. Cecile M. Ronckers ${ }^{1,2}$ t the DCOG-LATER Study Group}

1 Department of Pediatric Oncology, Emma Children's Hospital/Academic Medical Center, Amsterdam, The Netherlands

2 Princess Máxima Center for Pediatric Oncology, Heidelberglaan 25, 3584 CS Utrecht, The Netherlands
3 Dutch Childhood Oncology Group, Utrecht, The Netherlands

4 Department of Pediatric Oncology/Hematology, University Medical Center Groningen, University of Groningen, Groningen, The Netherlands 
5 Department of Pediatric Oncology/Hematology, Sophia Children's Hospital/Erasmus Medical Center, Rotterdam, The Netherlands

6 Department of Hematology, Radboud University Medical Center, Nijmegen, The Netherlands

7 Department of Internal Medicine/Endocrinology, Leiden University Medical Center, Leiden, The Netherlands

8 Department of Pediatric Oncology and Hematology, Wilhelmina Children's Hospital/University Medical Center Utrecht, Utrecht, The Netherlands
9 Department of Pediatric Oncology/Hematology, VU University Medical Center, Amsterdam, The Netherlands

10 Department of Registration, Netherlands Comprehensive Cancer Organisation, Utrecht, The Netherlands

11 Department of Radiation Oncology, University Medical Center Groningen, University of Groningen, Groningen, The Netherlands

12 Department of Epidemiology and Biostatistics, The Netherlands Cancer Institute, Amsterdam, The Netherlands 